\title{
Research on Reappearance Technique of Body Acceleration with the Frontal Crashworthiness Based On Modular Design
}

\author{
Jun-cheng Lv, Yong Wei, Yun-Jun Tang, Feng Wang \\ SAIC-GM-WULING Automobile Co., Ltd, Liuzhou \\ 545007, China \\ E-mail: juncheng.lv@sgmw.com.cn, \\ yongwei@sgmw.com.cn, yunjun.tang@sgmw.com.cn, \\ wangfeng95407@163.com
}

\begin{abstract}
Based on modular design, the micro-automobile body acceleration waveform reproduction technology has been studied. The design of equivalent structures trolley was to replace the mule cart during the development process of platform derivative models, and frontal crash test results showed that test methods can achieve reproducibility body acceleration, which can effectively improve the efficiency of research and development.
\end{abstract}

Keywords-micro-automobile; safe collisions; modular; body acceleration's reappearance; trolley test

\section{INTRODUCTION}

During the design and development process of new models, the body acceleration need to be adjusted to the target, Combined with the new collision boundary, verifying the new model body acceleration's waveform is key, when the key structural of the new model is optimized. verification of new models use numerical simulation as a reference, but there will be a problem of reliable accuracy; As for the frontal crash test about, the trial production cycle of sample vehicle is long: The change of the geometry size of energy absorption structures, such as longitudinal beam, will cause repetitive design, repetitive processing and small-batch manual assembly of related links parts.

In the study both at home and abroad, trolley is more applied to the process of validation test of Constraint system components, such as seat, trolley test aimed at structure always are applied to validation of energy absorption characteristics, whose effect is equivalent to the dynamic drop-weight test, domestic and foreign research are short of design of collision validation for The overall validation of body structure [1-5].

\section{THE EQUIVALENT SIMPLIFICATION OF BODY ACCELERATION WAVEFORM}

Due to the influence of deformation of energy absorption structure, body acceleration curve waveforms cannot directly measure the corresponding energy absorption index, such as Pm and SEA value, etc, to guide the optimization direction. This needs to make the acceleration waveform equivalent, simplified the waveform data we acquire will be more clearly reflect the energy absorption characteristics of the body structure.

As shown in Figure.1, Frontal crash acceleration

\author{
Yi-Min Mo, Feng Wang \\ School of Mechanical and Electrical Engineering, Wuhan \\ University of Technology, Wuhan, 430070, China \\ E-mail: moyimin@whut.edu.cn, \\ wangfeng95407@163.com
}

waveform of a mini-car is reduced to equivalent the second order wave, the six moment of A, B, C, D, E, F are the key point of collision, point A shows the moments when the body contact with rigid walls; point $\mathrm{B}$ shows the moments when Front longeron began to deform, anti-collision beam finished the deformation; point $\mathrm{C}$ shows the moments when the rigid body parts, such as engine and powertrain, contact with the walls; point $\mathrm{D}$ shows the moments when engine mounting fractured or Rear suspension structure deformed, this time can be measured through such a means as the test video and contact switch, the time from $\mathrm{C}$ to $\mathrm{D}$ is about 7-8 ms about the mini-car; vehicle speed is zero, rebound movement begin at $\mathrm{E}$ moment; the vehicle reached the maximum reverse speed at $\mathrm{F}$ moment.

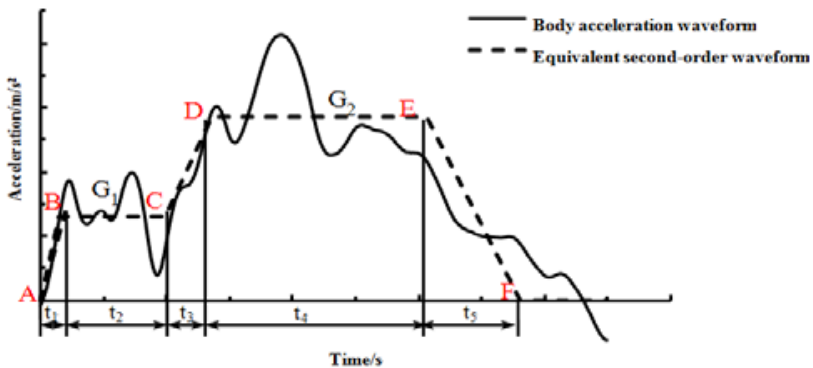

Figure 1. Body acceleration waveform in frontal crash and equivalent second-order waveform.

Equivalent second-order wave curve was composed of the equivalent acceleration for G1 and G2 and five time bucket of $\mathrm{t} 1$ to $\mathrm{t} 5$. The distance between the anticollision beam and motor head is $\mathrm{D} 1$, the time is $\mathrm{t} 1+\mathrm{t} 2$; the distance between the engine backend and firewall D2; firewall Intrusion is D3, as shown in Figure.2. The corresponding deformation D1 is SAC from A to C, corresponding distance of engine mounting failure is SCD from $C$ to $D, D-E$ is SDE. If the engine is changed in the derived model, SAC and SDE will change, but SCD fault distance of the engine mounting basically remain unchanged. 


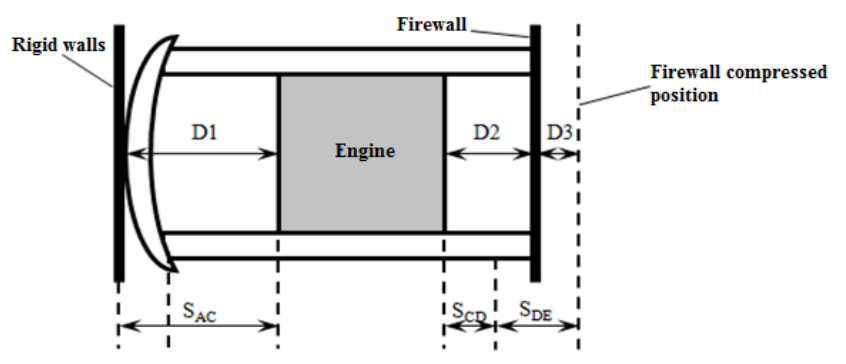

Figure 2. Deformation and energy absorption area of vehicle.

Based on conservation of energy, the calculation of the second order waveform which was simplified to equivalent needs to satisfy the following conditions[6], assuming S1 = SAC, S2 = SDE.

In order to verify the calculation accuracy of equivalent simplified second order wave, body acceleration waveform in frontal crash test of a vehicle should be calculated to compare, According to the experimental data, $\mathrm{S} 1=249.78 \mathrm{~mm}, \mathrm{SCD}=79.68, \mathrm{~S} 2=161.76$. By the formula 1 to 4 , equivalent waveform related parameters can be obtianed, $\mathrm{G} 1=130.47 \mathrm{~m} / \mathrm{s} 2, \mathrm{G} 2=285.07 \mathrm{~m} / \mathrm{s} 2, \mathrm{t} 1=2.9 \mathrm{~ms}, \mathrm{t} 2=16.9 \mathrm{~m} \mathrm{~s}$, $\mathrm{t} 3=7.2 \mathrm{~ms}, \mathrm{t} 4=34 \mathrm{~ms}, \quad \mathrm{t} 5=14.9 \mathrm{~ms}$. Body acceleration waveform and equivalent second-order waveform are shown in Figure.3.

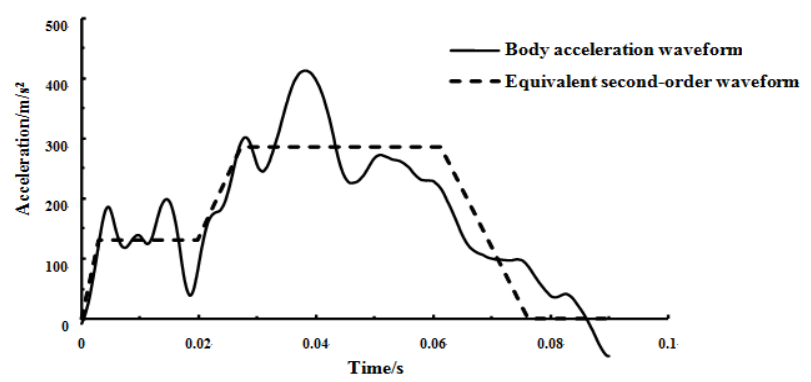

Figure 3. Body acceleration waveform in frontal crash and equivalent second-order waveform.

\section{EQUIVALENT DESIGN OF MICRO-AUTOMOBILE CRASH SAFETY STRUCTURE}

At the moment of vehicle collision, the load forces generated by the collision kinetic energy of the disperse to components connected through the rational design of each other topological relations between the load bearing structures. The reasonable design of intensity and rigidity makes the thin-walled beam of deformation zone engender sequential deformation to consume and absorb the collision kinetic energy, which will hit the mark of protecting the occupant safety. For the design of platform derivative vehicle models, if we want to make the mutual topological relations between the load bearing structures to change little while the maximum dynamic energy absorption distance to be changed (decrease) under conditions of meeting the agreement of occupant injury protection, we need to upgrade the energy-absorbing character of the energy-absorbing structures, which makes the force transmission path to change. As is shown in figure.4, in the allocation of the force transmission path of a certain type of Micro-Automobile, if the average crushing force of the former stringer increases, the small stringer section force then needs a corresponding increase as well to make sure the accordance of the engine compartment, that makes the entire transmission path to be changed not so much, and ensure that the firewall will not increase the amount of firewall intrusion because of the excessive force during crash.

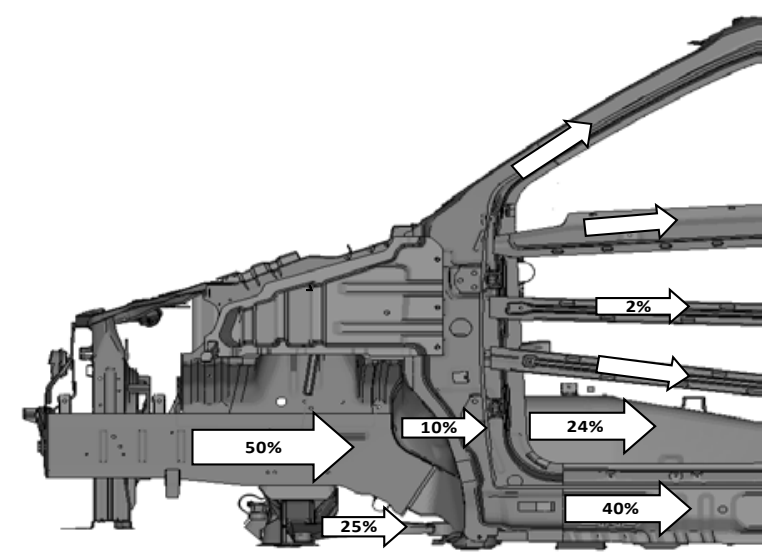

Figure 4. Allocation of the force transmission path of a certain type of Micro-Automobile.

There exists some structures have verified that trolley cannot meet the design requirements, as is shown in Figure.5 (a), the energy absorption characteristic of the front longitudinal beam corresponds to the magnitude of $G_{1}$ in the two order equivalent waveform, the original sled test can not be simulated for lack of car body stiffness and other structural $G_{2}$ value. The optimization design of the platform derived models lies in the relationship between the key section force and the structure space, when the maximum dynamic pressure collapse distance is changed, Compared with the previous, energy absorption zone will change, namely: the change of the distance between the engine and the firewall D2 affects the size of the $G_{2}$ value in the equivalent two - step waveform, considering vehicle design of simulation of car body stiffness and relative position of engine, as is shown in Figure.5 (b). 


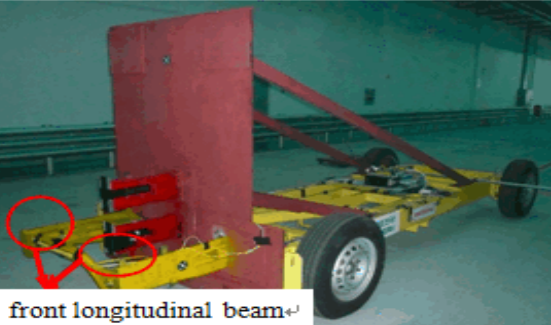

(a) the original structure verification trolley

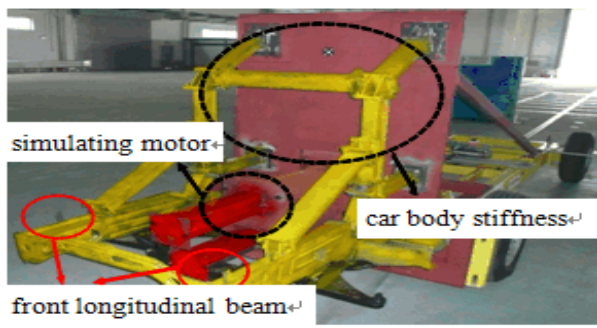

(b) structure validation equivalent trolley

Figure 5. Equivalent sled test design.

For a mini car, The distance between the engine and the firewall determines the value of D2, in the equivalent bogie structure, the engine and other dynamic components by the two thin-walled beams, the length of the upper thin wall is determined by the distance from the front end of the engine to the firewall, The mean crushing force is determined by the slope of the equivalent waveform Scd, The average compressive strength of the lower part of the thin-walled beam is determined by the force transfer distribution and the rigidity of the vehicle body, and the difference between the upper and the thin-walled beams is determined by the D2,
As shown in Figure.3 (b),thin-walled beam is in the two red labeled. The vehicle body structure is corresponding to the thin wall beam of the trolley deformation energy absorbing area. The simulation of the car body stiffness needs to consider the force transmission of the deformation structure in the frontal impact, five parts of small wing longeron / engine compartment cover, engine / gearbox / tyre, a column, threshold / front door / the top structure of the car , wheel cover / cowl panel are designed the equivalent of thin-walled beam structure corresponding to collision force, as is shown in Figure.4.

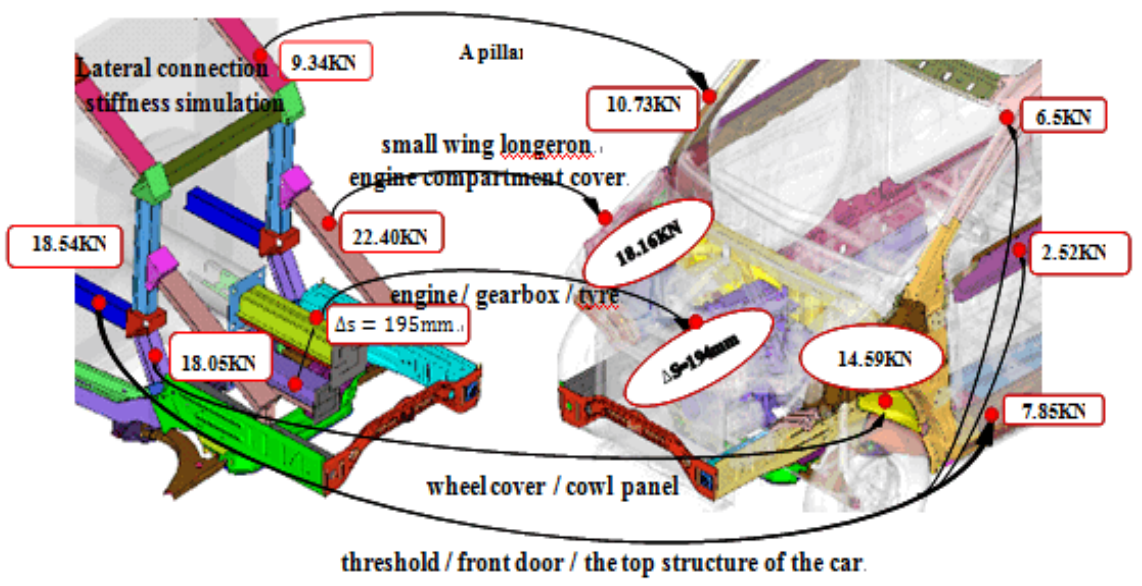

Figure 6. Corresponding relationship between the equivalent structure of a certain type of mini vehicle.

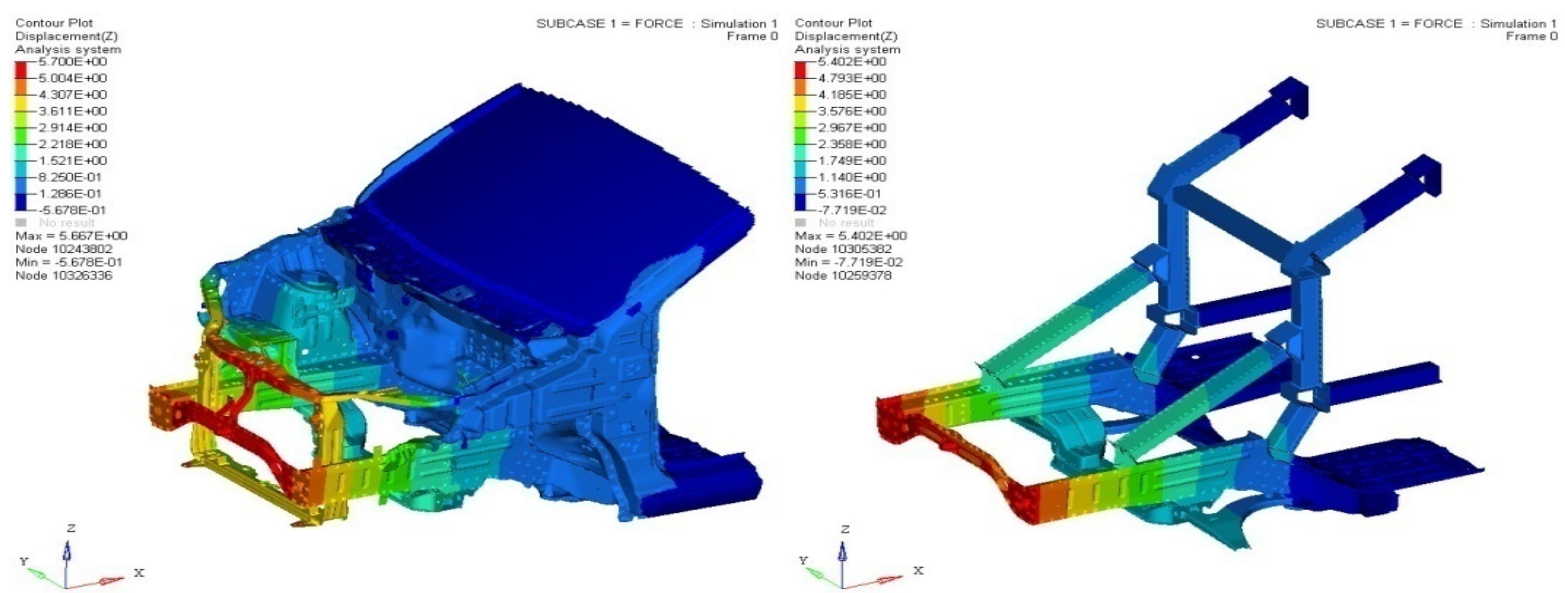

Figure 7. Deformation cloud image of the vehicle and the equivalent trolley. 
In Figure.6, the equivalent compressive strength of thin-walled beam of equivalent structure is obtained from the body structure, the structure size is determined by the relative position of the body structure, the average crushing force can be designed with the characteristics of energy absorption in the previous paper, In this paper, the deformation form of single hat - shaped thin - walled beam is more stable, and the design of thin-walled beam is single hat cross section. Each connecting joint is welded by steel plate, The bottom of the beam is welded on the bottom plate, and the bottom plate is connected with the joint by a bolt, and the form is simple and convenient to disassemble.

In the matching process of the car body stiffness and the equivalent structure, considering the complexity of the whole vehicle structure, more and more parts of the trolley can be ignored, in order to get a better structural form, the stiffness simulation model is established for the equivalent part of each stiffness after determining the key structure of the energy absorbing member equivalent thin-walled beam, Through the stiffness comparison, the size of the equivalent stiffness of thin-walled beam is optimized, and the overall stiffness of the upper body is consistent with the car structure. The loading force distance of the top of the front longitudinal beam is $50 \mathrm{~mm}, 50 \mathrm{kN}$ force in vertical upward direction is applied to left and right longitudinal beams, in order to prevent the local deformation from affecting the whole stiffness, the loading area is $50 * 50 \mathrm{~mm}$; The interception distance from stringer tip of vehicle and the trolley is $1850 \mathrm{~mm}$, and rigid fixed connection.

The calculation results show that the maximum displacement of the equivalent structure of the whole vehicle and the trolley is 5.67 and $5.40 \mathrm{~mm}$, deformation nephogram is shown in Figure.7. The stiffness error of the two structures is less than $5 \%$, which shows that the stiffness of the structure is equivalent to the equivalent.

Using equivalent average crushing force design thin-walled beam section of the equivalent structure of the trolley, Stiffness model is established to calculate the

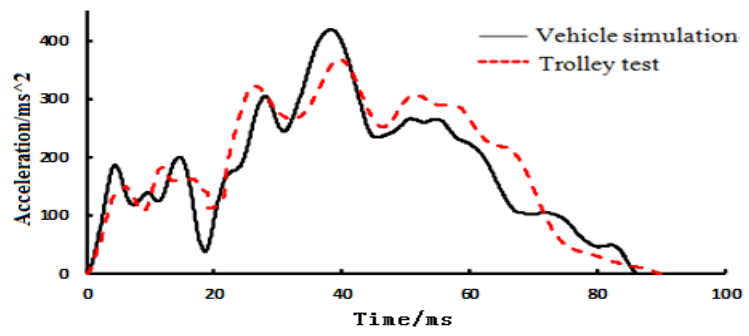

stiffness error between the structure, and the thin-walled beams of no energy absorption are optimized, equivalent structure effect is good that be proved by stiffness calculation results.

\section{EQUIVALENT TROLLEY BODY}

\section{ACCELERATION EMERSION VALIDATION TEST}

To verify the feasibility of equivalent structure and trolley body acceleration repetition, trolley prototype trial-manufacture in accordance with the equivalent simulation model structure design and manufacture,as shown in Figure.5 (b), equivalent thin-walled beam of A post, longitudinal beam and the auxiliary frame and the connection of equivalent thin-walled beam of engine power assembly and the homemade mounting plate of side impact, to collision with rigid walls by $50 \mathrm{~km} / \mathrm{h}$. To collect acceleration signal through three road that left and centre and right of the trolley, sampling frequency of $1 \mathrm{KHZ}$, in the thin-walled beam of equivalent engine power have touch switch $\mathrm{t} 3$ to measure the time of touch. Trolley compared with deformation of the vehicle frontal crash simulation, as shown in Figure.8, test site for China automotive engineering research institute (chongqing) collision safety center front collision testing ground.
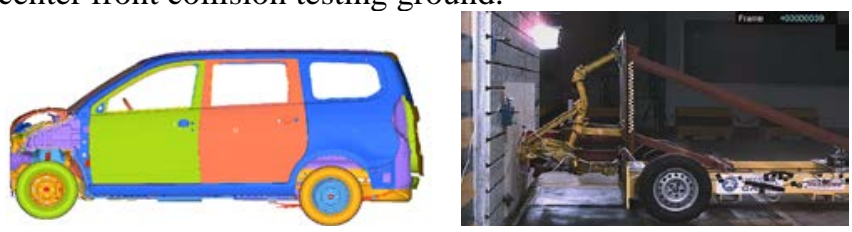

Figure 8. Vehicle simulation compared with the equivalent deformation of trolley.

Equivalent trolley test results with the vehicle simulation results contrast, known vehicle speed and acceleration waveform consistency, the key point is basic coincidence such as the time of speed is zero, as shown in Figure.9.

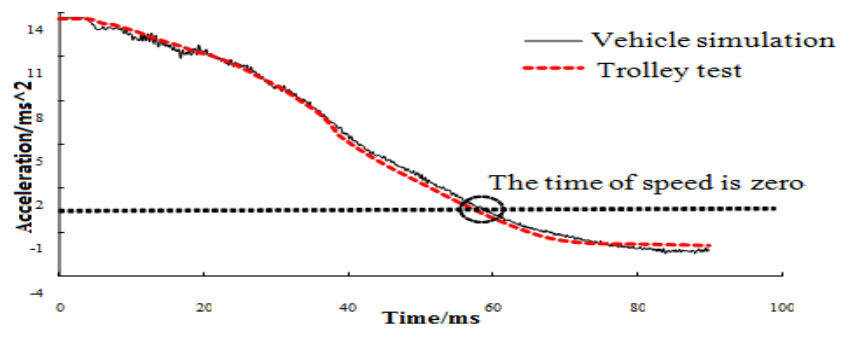

Figure 9. Vehicle model compared with test speed/acceleration waveform of trolley.

The frontal crash test of equivalent structure trolley compared with the vehicle simulation results, known acceleration waveform consistency, equivalent acceleration G1 is $134 \mathrm{~m} / \mathrm{s} 2$ and $130.47 \mathrm{~m} / \mathrm{s} 2, G 2$ is $281.4 \mathrm{~m} / \mathrm{s} 2$ and $285.07 \mathrm{~m} / \mathrm{s} 2$, the time that speed is zero is $61.3 \mathrm{~ms}$ and 62.7 ms separately, it is shown that equivalent structure trolley replace mule cart can make the acceleration waveform come true again.

In the process of the establishment of the platform derivative simulation model, the finite element model of the mule cart have a total of 12 kinds of materials, 1162187 nodes, 1074871 shell elements, as well as 1074871 individual units, use the LS - DYNA971 solver in DELL T7600 workstation (32 threads, $2.7 \mathrm{GHz}, 64 \mathrm{~g}$ RAM) need to be more than 6 hours; equivalent simulation model of trolley structure have 8 kinds of materials, 216425 shell elements, 8325 individual units, computation time is 1.2 hours. Calculation model of equivalent structure trolley can dramatically improve the efficiency of optimization, as a validation of structure optimization mode to replace vehicle 
model.

\section{CONCLUSION}

considering the mule cart faults of manufacturing high cost and low efficiency, using the concept of equivalent to built the frontal crash model of equivalent structure trolley to verifying the rationality of acceleration, vehicle test show that the equivalent structure can achieve the mule cart frontal crash test;

The frontal crash test of equivalent structure trolley compared with the vehicle simulation results, known acceleration waveform consistency, equivalent acceleration G1 is $134 \mathrm{~m} / \mathrm{s} 2$ and $130.47 \mathrm{~m} / \mathrm{s} 2, \mathrm{G} 2$ is $281.4 \mathrm{~m} / \mathrm{s} 2$ and $285.07 \mathrm{~m} / \mathrm{s} 2$, the time that speed is zero is $61.3 \mathrm{~ms}$ and 62.7 ms separately, it is shown that equivalent structure trolley replace mule cart can make the acceleration waveform come true again.

\section{REFERENCES}

[1] Zaouk A K ,Marzougui D, Kan C D . Development of a Detailed Vehicle Finite Element Model Part II : Material Characterization and Component Testing [J] . International Journal of Crashworthiness, 2000, 5 (1): 37-50.

[2] Kirkpatrick S W. Development and validation of high fidelity vehicle crash simulation models[R]. SAE Technical Paper, 2000.

[3] Dazhi Wang, Fanzhong Kong, Shilin Huang, et al. Improvement Design of Minibus' s Frame Structure in Frontal Impact [J] . Journal of Highway and Transportation Research and Development, $2004,21(2): 109-122$.

[4] Liqiang Dong, Guangyao Li, Tao Chen, Hui Gao, et al. A NewMethod of Simplified Modelling for Atuomobile Frontal Impact Sled [J]. China Mechanical Engineering,2009,21(12): 1507-1511.

[5] Libo Cao,Peigang Yan. Reproduce of Vehicle Frontal Crash Waveform Using Thin-walled Circular Energy Absorption Tube [C]. The 8th Int. Forum of Automotive Traffic Safety (INFATS), December 2010, Wuhu, China.

[6] Zhixiong Ma, Xichan Zhu. An Analysis on the Sensitivity of Main Dummy In jury Criteria to Equivalent Dual-trapezoid Deceleration Curve [J]. Automotive Engineering,2009,31(2): 165-169. 